\title{
AN INVESTIGATION OF ELECTROMAGNETIC WAVE PROPAGATION IN PLASMA BY SHOCK TUBE*
}

\author{
ZHU Naiyi (竺乃宜) ${ }^{\dagger}$ LI Xuefen (李学芬) HUANG Lishun (黄立舜) \\ YU Xilong (余西龙) YANG Qiansuo(杨乾锁) \\ (Laboratory of High Temperature Gas Dynamics, Institute of Mechanics, Chinese Academy of Sciences, \\ Beijing 100080, China)
}

\begin{abstract}
This paper presents the electromagnetic wave propagation characteristics in plasma and the attenuation coefficients of the microwave in terms of the parameters $n_{e}, v, \omega, L, \omega_{b}$. The $\phi 800 \mathrm{~mm}$ high temperature shock tube has been used to produce a uniform plasma. In order to get the attenuation of the electromagnetic wave through the plasma behind a shock wave, the microwave transmission has been used to measure the relative change of the wave power. The working frequency is $f=(2 \sim 35) \mathrm{GHz}(\omega=2 \pi f$, wave length $\lambda=15 \mathrm{~cm} \sim 8 \mathrm{~mm})$. The electron density in the plasma is $n_{e}=\left(3 \times 10^{10} \sim 1 \times 10^{14}\right) \mathrm{cm}^{-3}$. The collision frequency $v=\left(1 \times 10^{8} \sim 6 \times 10^{10}\right) \mathrm{Hz}$. The thickness of the plasma layer $L=(2 \sim 80) \mathrm{cm}$. The electron circular frequency $\omega_{b}=e B_{0} / m_{e}$, magnetic flux density $B_{0}=(0 \sim 0.84) \mathrm{T}$. The experimental results show that when the plasma layer is thick (such as $L / \lambda \geq 10$ ), the correlation between the attenuation coefficients of the electromagnetic waves and the parameters $n_{e}, v, \omega, L$ determined from the measurements are in good agreement with the theoretical predictions of electromagnetic wave propagations in the uniform infinite plasma. When the plasma layer is thin (such as when both $L$ and $\lambda$ are of the same order), the theoretical results are only in a qualitative agreement with the experimental observations in the present parameter range, but the formula of the electromagnetic wave propagation theory in an uniform infinite plasma can not be used for quantitative computations of the correlation between the attenuation coefficients and the parameters $n_{e}, v, \omega, L$. In fact, if $\omega<\omega_{p}, v^{2} \ll \omega^{2}$, the power attenuations $K$ of the electromagnetic waves obtained from the measurements in the thin-layer plasma are much smaller than those of the theoretical predictions. On the other hand, if $\omega>\omega_{p}, v^{2} \ll \omega^{2}$ (just $v \approx f$ ), the measurements are much larger than the theoretical results. Also, we have measured the electromagnetic wave power attenuation value under the magnetic field and without a magnetic field. The result indicates that the value measured under the magnetic field shows a distinct improvement.
\end{abstract}

KEY WORDS: microwave, plasma, shock tube

\section{INTRODUCTION}

A great attention has been paid to the electromagnetic wave propagation characteristics in plasma because of its important engineering applications. There has been a reasonable theoretical model ${ }^{[1,2]}$ in the infinite uniform and non-electromagnetic field plasma, but it is not quite valid in the thin layered plasma. Besides, the nature of the plasma and the characteristics of the electromagnetic wave propagation in the plasma will be changed owing to the additional magnetic field. In light of the correlation between the electromagnetic wave working frequency $\omega$, the natural frequency of electron in plasma $\omega_{p}$, the collision frequency $v$, the electron cyclotron frequency $\omega_{b}$ and the thickness $L$ of the plasma sheath, the quantitative study of the electromagnetic wave propagation along the magnetic field direction in an

Received 2 July 2002, revised 8 April 2003

* The project supported by the National Natural Science Foundation of China (19982005 and 10032050) and the National Defense Science Foundation of China

† E-mail: zny@imech.ac.cn 
anisotropic plasma will be very difficult, and the experimental research becomes very important.

\section{ELECTROMAGNETIC WAVE INTERAC- TION WITH PLASMA}

It is assumed that the plasma consists of equal numbers of positive ions and free electrons together with a number of neutral particles. The charged particles maintain an average equilibrium separation distance due to their electrostatic fields. If one of the charged particles is displaced from its equilibrium position and the other charges remain fixed, the displaced charge will oscillate about its equilibrium position in a manner of a mass on a spring. The particle is the mass, the restoring electrostatic force due to the neighboring charged particles is the spring, and the collisions of the oscillating charge with the neutral particles constitute the damping. The frequency of oscillation of the charged particle is called the plasma frequency. It is the natural frequency of a free charge in a plasma. The natural frequency $\omega_{p}$ for electrons in the plasma is defined by the relation

$$
\omega_{p}=\left(n_{e} e^{2} / \varepsilon_{0} m_{e}\right)^{1 / 2}
$$

where $n_{e}$ is the number of electrons per unit volume, $-e$ is the electronic charge, $m_{e}$ is the mass of the electron, and $\varepsilon_{0}$ is the permittivity of the free space. Since the ion mass is about four orders of magnitude larger than the electron mass, the ion plasma frequency is much smaller than the electron plasma frequency for a given plasma.

In the preceding example the electron was displaced from its equilibrium position and was allowed to oscillate at its natural frequency in the plasma. An electromagnetic wave, however, acts as a periodic driving force on the electron. If the driving frequency (i.e., the electromagnetic wave frequency) is considerably less than the natural frequency of the plasma electron and if the collision damping of the electron motion is small, the inertial effects are small and the electron will oscillate at the driving frequency. The oscillating charge acts as a dipole radiator, producing both a forward-traveling and a backward-traveling electromagnetic wave. The backward-traveling wave appears as a reflected wave while the forward-traveling wave is out of phase with the driving signal and tends to cancel it. This process is repeated as the driving signal penetrates the plasma, resulting in an attenuation of the driving signal, which increases with the thickness of the plasma.

The situation is completely changed when the frequency of the electromagnetic wave is much greater than the electron plasma frequency. The electron now exhibits large inertial effects and oscillates only weakly at the driving frequency. As a result, the electromagnetic wave propagates unattenuated if no electron collisions occur. A slight reflection and attenuation of the electromagnetic wave is experienced if electron collisions do occur.

The wave equation satisfied by the electric field vector $E$ for the interaction between electromagnetic waves and plasma in a homogeneous isotropic sourcefree medium is ${ }^{[1]}$

$$
\nabla^{2} \boldsymbol{E}-\mu \sigma \frac{\partial \boldsymbol{E}}{\partial t}-\mu \varepsilon \frac{\partial^{2} \boldsymbol{E}}{\partial^{2} t}=0
$$

where $\mu$ is the permeability, $\sigma$ is the conductivity, $\varepsilon$ is the permittivity, $\sigma=\varepsilon_{0} \omega_{p} /(v+\mathrm{i} \omega)\left(v^{2}+\omega^{2}\right)$, $\varepsilon=\left(K_{r}+\mathrm{i} K_{i}\right) \varepsilon_{0}, K_{r}=X Z /\left(1+Z^{2}\right), X=\omega_{p}^{2} / \omega^{2}$, $Z=v / \omega$, and $v$ is the electron collision frequency, $\omega$ is the electromagnetic wave frequency. One-dimensional plane-wave solutions of Eq.(2) are of the form ${ }^{[2]}$

$$
\boldsymbol{E}=\boldsymbol{E}_{0} \exp [ \pm(\alpha-\mathrm{i} \beta) x]
$$

where $\boldsymbol{E}_{0}$ is a vector constant. The attenuation coefficient $\alpha$ and the phase coefficient $\beta$ are defined as

$$
\begin{aligned}
& \alpha=K_{0}\left\{\frac{1}{2}\left[\left(K_{r}^{2}+K_{i}^{2}\right)-K_{r}\right]\right\}^{1 / 2} \\
& \beta=K_{0}\left\{\frac{1}{2}\left[\left(K_{r}^{2}+K_{i}^{2}\right)+K_{r}\right]\right\}^{1 / 2}
\end{aligned}
$$

When the electronic density in the plasma increases, $K_{r}$ and $\alpha$ will increase in general, that is to say, the attenuation of the electromagnetic wave will increase during the propagation.

When there is a static magnetic field in the plasma, with the coordinates so chosen that the magnetic field is in the $z$ direction, the complex dielectric coefficients of the plasma $(K)$ will be given by ${ }^{[3]}$

$$
K=\left|\begin{array}{ccc}
\varepsilon_{11} & \mathrm{i} \varepsilon_{12} & 0 \\
-\mathrm{i} \varepsilon_{12} & \varepsilon_{11} & 0 \\
0 & 0 & \varepsilon_{33}
\end{array}\right|
$$

where $\varepsilon_{11}=1+X(1-\mathrm{i} Z) /[Z+\mathrm{i}(1-Y)][Z+\mathrm{i}(1+Y)]$, $\varepsilon_{12}=X Y /[Z+\mathrm{i}(1-Y)][Z+\mathrm{i}(1+Y)], \quad \varepsilon_{33}=$ $1-X /(1+\mathrm{i} Z), Y=\omega_{b} / \omega, \omega_{b}=e B_{0} / m_{e}, \omega_{b}$ is the electron cycilotron frequency, $B_{0}$ is the magnetic flux density. The free electrons take a circular motion with $\omega_{b}$ in a plane perpendicular to the magnetic line. If the $B_{0}$ is large enough, the cyclotron radius of electrons will be very small. It means that the electrons will be 
frozen around the magnetic line and has no effect on the plane electromagnetic wave, which is perpendicular to $B_{0}$ in front of the wave. The above condition is advantageous for the electromagnetic wave to penetrate the plasma. For the propagation of plane waves in the uniform plasma along the direction of the static magnetic field, the attenuation coefficient $\alpha$ and the phase coefficient $\beta$ for the electromagnetic fields are

$$
\begin{aligned}
& \alpha_{ \pm}=K_{0}\left\{\frac{1}{2}\left[\left(K_{r \pm}^{2}+K_{i \pm}^{2}\right)-K_{i \pm}\right]\right\}^{1 / 2} \\
& \beta_{ \pm}=K_{0}\left\{\frac{1}{2}\left[\left(K_{r \pm}^{2}+K_{i \pm}^{2}\right)+K_{r \pm}\right]\right\}^{1 / 2}
\end{aligned}
$$

where $K_{r \pm}=1-X(1 \pm Y) /\left[\left(1 \pm Y^{2}\right)+Z^{2}\right], K_{i \pm}=$ $X Z /(1 \pm Y)^{2}$, the subscript + represents the lefthand circularly polarized wave, the subscript - represents the right-hand circularly polarized wave. From above formula, for the left-hand circularly polarized wave, if the magnetic flux density $B_{0}$ increases, $Y$ increases and $\alpha_{+}$decreases. In general, for the righthand circularly polarized wave, if $B_{0}$ and $Y$ are large enough, the attenuation coefficient $\alpha_{-}$will rapidly decrease.

\section{EXPERIMENTAL EQUIPMENT}

The uniform plasma is obtained from the experimental section of the shock tube ${ }^{[4]}$ and the transmitting antenna is fixed on the Teflon window in the sidewall of the experimental section. The electromagnetic wave enters in this section through the window and interacts with the shell layer of the plasma, and then the wave passes through the symmetric Teflon window in the lower sidewall of the experimental section and is received by a receiving antenna at the bottom of this section, as shown in Figs.1 3.

The superconductor coil fixed in the Dewar bottle is arranged around the transmitting antenna. The magnetic field direction of the coil center coincides with the propagation direction of the electromagnetic wave or in its opposite direction, but the magnetic field direction is perpendicular to the flow direction of the plasma.

By using an electric probe in the sidewall of the shock tube to trigger the recorder TCG8000 through an amplifer M113, one may guarantee the synchronous operation with the plasma in the shock tube.

The microwave signal is in a continuous working status. When the plasma behind the shock wave reaches the window in the experimental section, the electromagnetic wave will attenuate. If there is a strong magnetic field in this time, the attenuation level of the electromagnetic wave is different from that with no magnetic field. The records of these two experiments of the same parameters with and without magnetic field are compared, and the influence of the magnetic field to the electromagnetic wave propagation characteristics can be seen.

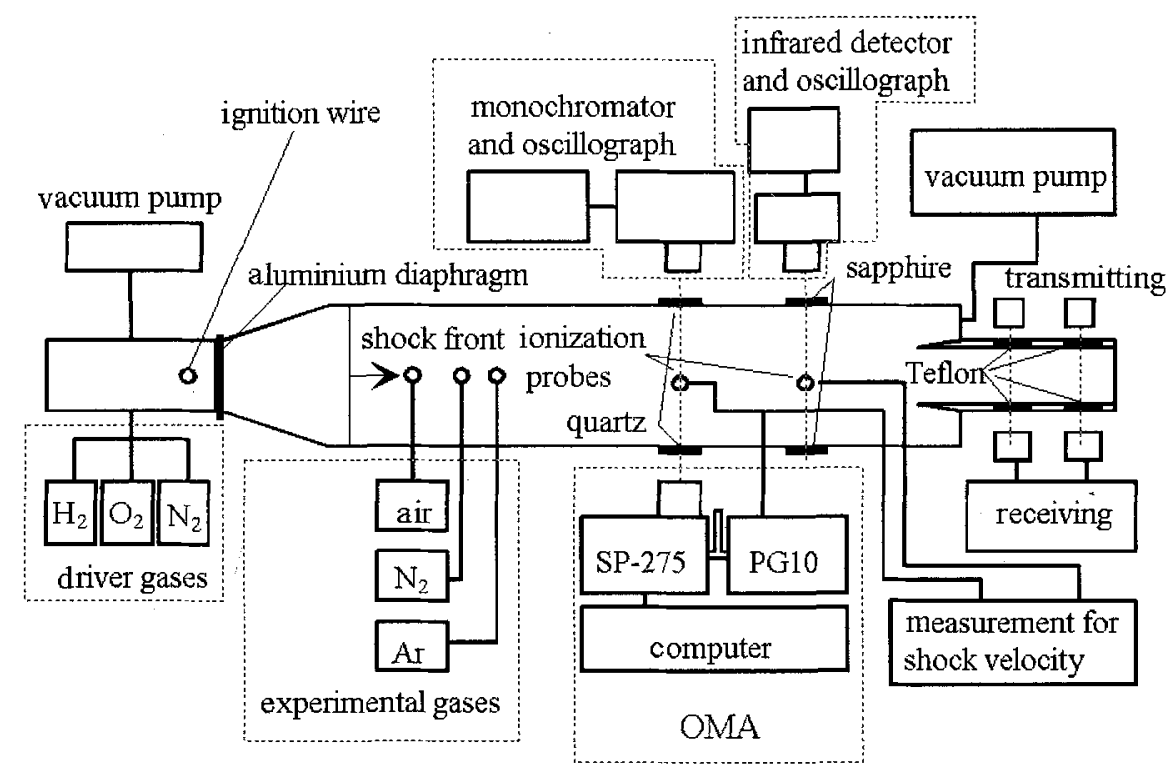

Fig.1 The experiment setup of $\phi 800 \mathrm{~mm}$ shock tube and the measurement instruments 


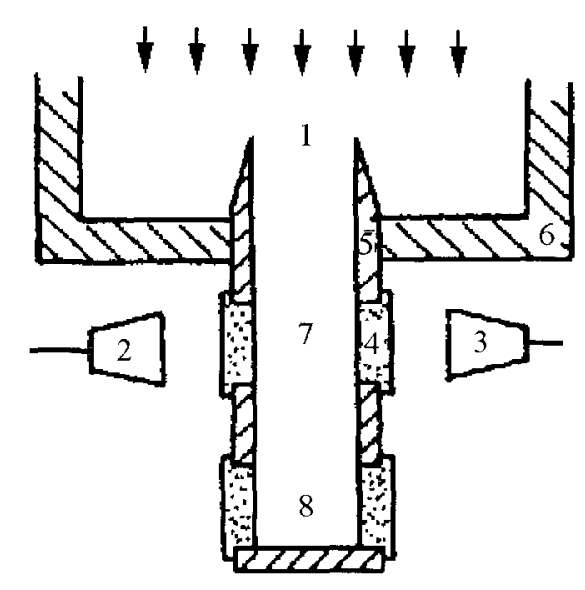

$$
\begin{array}{ll}
1 \text { - plasma flow } & 2-\text { transmitting antenna } \\
3 \text { - receiving antenna } & 4 \text { - window with Teflon } \\
5-\text { test section wall } & 6 \text { - shock tube wall } \\
7 \text { - incident shock wave region } & 8 \text { - reflected shock wave region }
\end{array}
$$

Fig.2 The experimental arrangement

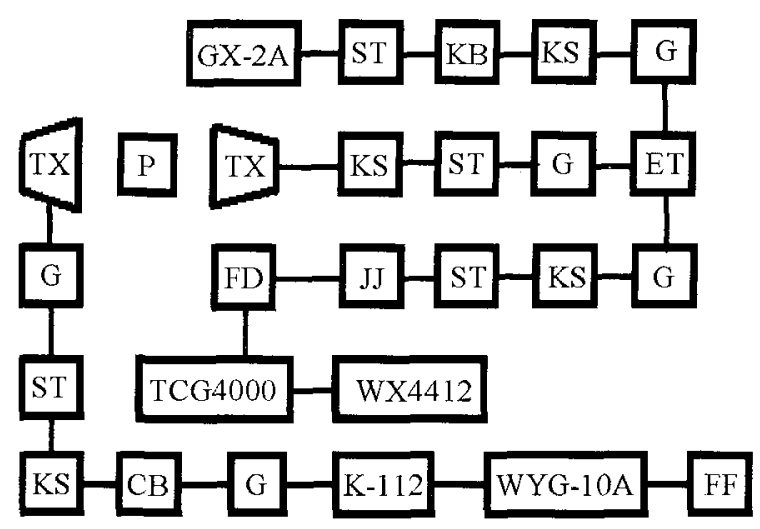

$\mathrm{FF}$ - square wave generator

K-112 - klystron

$\mathrm{CB}$ - long wave guide

$\mathrm{ST}$ - impedance matching unit

$\mathrm{P}$ - plasma

$\mathrm{KB}$ - frequency meter

$\mathrm{JJ}$ - crystal detector

TCG4000 - transient converter
WYG-10A - klystron power

$\mathrm{G}$ - segregator

$\mathrm{KS}$ - pad attenuator

TX - antenna

ET, E-T - wave guide connection

GX-2A - power tester

FD - resistance load

WX4412 - recorder

Fig.3 The block diagram of microwave transmission

The signal of the microwave from the detector is sampled and stored by a transient converter TCG8000. The oscilloscope SBM-14 is used to continuously display the signal. Another way is to use a plotter WX4412 to record the plot.

\section{CONDITION PARAMETERS}

\subsection{Electronic Density $n_{e}$}

The electron density behind normal shock waves is measured systematically, over the range of $P_{1}=$ $(1.33 \sim 133) \mathrm{Pa}, M_{S}=9 \sim 22.5$, by the use of the near-free-molecular Langmuir probes, the ordinary microwave transmission, the special highly sensitive microwave transmission and microwave reflection methods, and the microwave interferometer, made by the authors. By using our data, an approximate equation $n_{e} \propto P_{1}$ is obtained for constants $M_{S}$. This approximate equation gives results that agree with the theoretical predictions ${ }^{[5 \sim 7]}$.

\subsection{Collision Frequency $v$}

The collision frequency in the plasma can be expressed as ${ }^{[6]}$ 


$$
v=v_{\mathrm{e}}+v_{\mathrm{en}}
$$

The collision frequency of electron with positive ion is

$$
v_{\mathrm{ei}}=\left(8 K T / \pi m_{e}\right)^{1 / 2} n_{i} \cdot Q_{\mathrm{ei}}
$$

where $K$ is the Boltzmann constant, $m_{e}$ is the mass of electron, $n_{i}$ is the density of the positive ion number, $Q_{\mathrm{ei}}$ is the collision section of electron with positive ion. When the level of ionization is small, $v_{\mathrm{ei}} \ll v_{\mathrm{en}}$ in general, and an approximate theoretical equation may be used as

$$
Q_{\mathrm{ei}}=\left(20.2 \times 10^{-6}\right) \lg \left(221 T / n_{e}^{1 / 3}\right)
$$

The collision frequency of electron with neutral particle

$$
v_{\mathrm{en}}=\left(8 K T / \pi m_{e}\right)^{1 / 2} n_{i} \cdot Q_{\mathrm{en}}
$$

where $Q_{\mathrm{en}}$ is the average collision section of electron with neutral particle and can be determined by experiments ${ }^{[8]}$ or theoretical calculations.

\subsection{Magnetic Flux Density $B_{0}$}

The magnetic flux density caused by the superconductor coil is not only proportional to the local current, but also bears a relation with the environment near the Dewar bottle and the experimental section. In the experimental region of the $\phi 800 \mathrm{~mm}$ shock tube, the magnetic flux density is about 0.01 $\mathrm{T} / \mathrm{A}$, as measured by the magnetic probe in the center of Dewar bottom. The magnetic flux density in the center of the experimental section in this test is about $0.0068 \mathrm{~T} / \mathrm{A}$ by the theoretical calculation.

\subsection{Electromagnetic Wave Attenuation Characteristics}

In order to acquire the exact power attenuation of the microwave in different plasma shells and to improve the power attenuation by a strong magnetic field, a precise attenuator HEWLETT PACKARD is used to give a static demarcation for the attenuation characteristic curve. The attenuator is composed of a series connection of $8496 \mathrm{~B}$ ATTENUATOR/110 dB and $8494 \mathrm{~B}$ ATTENUATOR/11 dB and has a working frequency range of $\mathrm{DC}-18 \mathrm{GHz}$. This attenuator is put in series in the actual circuit of this test in order to simulate the power attenuation caused by plasma. In the condition of each grade from $0 \mathrm{~dB}$ to $25 \mathrm{~dB}$, the electric wave signal, which is acquired from TCG8000, may be plotted by WX4412 plotter to show the effective amplitude value. If we let the amplitude be equal to one for $0 \mathrm{~dB}$ and then the other value of amplitude in various $\mathrm{dB}$ may be obtained.
A curve (a static demarcation curve) for the relative amplitude value of the electromagnetic wave against the power attenuation value is obtained. Every sheet of the electromagnetic wave signal pattern is recorded in the experiment. Based on the relative attenuation amplitude value in the actual measurement, the corresponding power attenuation is very easy to be read in light of the demarcation curve.

\section{EXPERIMENTAL RESULTS}

The experimental results show that: when the plasma layer is thick (such as $L / \lambda \geq 10$ ), the correlation between the attenuation coefficients of the electromagnetic waves and the parameters $n_{e}, v, \omega, L$ determined from the measurements are in good agreement with the theoretical predictions for electromagnetic wave propagations in the uniform infinite plasma. (Figs.4 and 5, Tables 1 3).

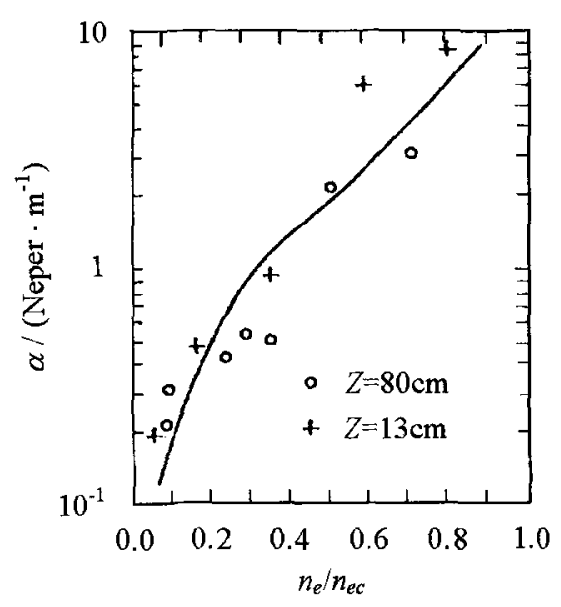

Fig.4 $8 \mathrm{~mm}$ microwave

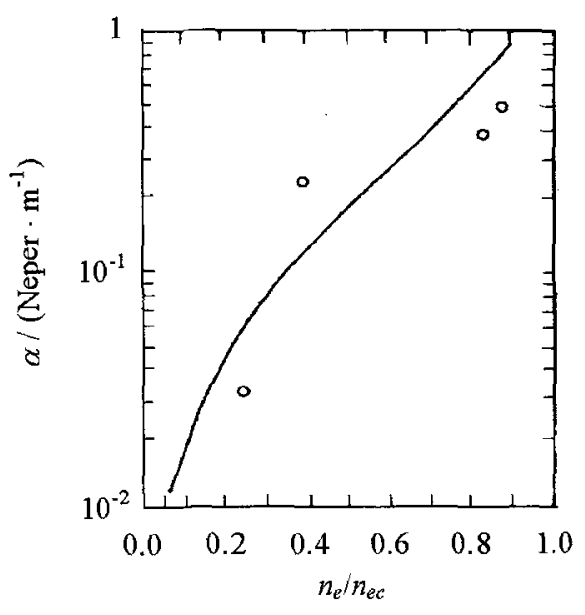

Fig. $53 \mathrm{~cm}$ microwave 
Table 1 Influence of $v$ on $\alpha$

\begin{tabular}{ccccc}
\hline$n_{e} / \mathrm{cm}^{-3}$ & $Z / \mathrm{cm}$ & $f / \mathrm{GHz}$ & $v / \mathrm{Hz}$ & $\alpha /\left(\right.$ Neper $\left.\cdot \mathrm{m}^{-1}\right)$ \\
\hline $1.9 \times 10^{12}$ & 80 & 35.5 & $1.0 \times 10^{10}$ & 2.9 \\
$1.6 \times 10^{12}$ & 80 & 35.5 & $1.1 \times 10^{9}$ & 0.31 \\
\hline
\end{tabular}

Table 2 Influence of $\lambda$ on $K$

\begin{tabular}{ccccc}
\hline$n_{e} / \mathrm{cm}^{-3}$ & $v / \mathrm{Hz}$ & $Z / \mathrm{cm}$ & $\lambda / \mathrm{cm}$ & $K / \mathrm{dB}$ \\
\hline $2.8 \times 10^{12}$ & $3 \times 10^{8}$ & 80 & 3 & 20 \\
$2.8 \times 10^{12}$ & $3 \times 10^{8}$ & 80 & 0.8 & 1 \\
\hline
\end{tabular}

Table 3 Influence of $Z$ on $K$

\begin{tabular}{ccccc}
\hline$n_{e} / \mathrm{cm}^{-3}$ & $v / \mathrm{Hz}$ & $\lambda / \mathrm{cm}$ & $Z / \mathrm{cm}$ & $K / \mathrm{dB}$ \\
\hline $1.1 \times 10^{13}$ & $1.7 \times 10^{9}$ & 0.8 & 3.3 & 1 \\
$1.1 \times 10^{13}$ & $1.7 \times 10^{9}$ & 0.8 & 13 & 10 \\
$1.1 \times 10^{13}$ & $1.7 \times 10^{9}$ & 0.8 & 80 & 20 \\
\hline
\end{tabular}

When the plasma layer is thin (such as when both $L$ and $\lambda$ are of the same order), the theoretical results are only in a qualitative agreement with the experimental observations in the present parameter range, but the formula of the electromagnetic wave propagation theory in a uniform infinite plasma can not be used for quantitative computations of the correlation between the attenuation coefficients and the parameters $n_{e}, v, \omega, L$. In fact, if $\omega<\omega_{p}$, $v^{2} \ll \omega^{2}$, the power attenuations $K$ of the electromagnetic waves obtained from the measurements in the thin-layer plasma are much smaller than that of the theoretical predictions (Table 4). On the other hand, if $\omega>\omega_{p}, v^{2} \ll \omega^{2}$ (just $v \approx f$ ), the measurements are much larger than the theoretical results (Table 5).

Table 4 Comparison of experiment and Eq.(4)

\begin{tabular}{cccccc}
\hline$F / \mathrm{GHz}$ & $n_{e} / \mathrm{cm}^{-3}$ & $v / \mathrm{Hz}$ & $L / \mathrm{cm}$ & $K_{\exp } / \mathrm{dB}$ & $K_{\text {Eq. }(4)} / \mathrm{dB}$ \\
\hline 9.178 & $1 \times 10^{13}$ & $2 \times 10^{9}$ & 4 & 3 & 210 \\
9.178 & $8 \times 10^{12}$ & $1.5 \times 10^{9}$ & 6 & 1 & 280 \\
9.178 & $4 \times 10^{12}$ & $3 \times 10^{9}$ & 6 & 4.4 & 200 \\
5.92 & $8 \times 10^{12}$ & $1.5 \times 10^{9}$ & 6 & 3 & 280 \\
\hline
\end{tabular}

Table 5 Comparison of experiment and Eq. (4)

\begin{tabular}{cccccc}
\hline F/GHz & $n_{e} / \mathrm{cm}^{-3}$ & $v / \mathrm{Hz}$ & $L / \mathrm{cm}$ & $K_{\exp } / \mathrm{dB}$ & $K_{\mathrm{Eq} \cdot(4)} / \mathrm{dB}$ \\
\hline 9.178 & $5 \times 10^{11}$ & $8 \times 10^{9}$ & 6 & 30 & 3.4 \\
9.178 & $5 \times 10^{11}$ & $8 \times 10^{9}$ & 6 & 30 & 3.4 \\
9.178 & $3 \times 10^{11}$ & $7 \times 10^{9}$ & 4 & 2.7 & 0.12 \\
9.178 & $9 \times 10^{11}$ & $3 \times 10^{9}$ & 4 & 2 & 0.15 \\
\hline
\end{tabular}

Meanwhile, we investigate the propagation characteristics when $3.9 \mathrm{GHz}$ microwave penetrates through the thin plasma layer under strong magnetic fields and two states $\left(n_{e} \approx 3 \times 10^{13} \mathrm{~cm}^{-3}, v \approx\right.$
$9 \times 10^{9} \mathrm{~Hz}, B_{0} \approx(0 \sim 0.84) \mathrm{T} ; n_{e} \approx 4 \times 10^{12} \mathrm{~cm}^{-3}$, $\left.v \approx 2.4 \times 10^{9} \mathrm{~Hz}, B_{0} \approx(0 \sim 0.84) \mathrm{T}\right)$ are tested. Also, we measure the electromagnetic wave power attenuation value under the magnetic field and without a magnetic field. The result indicates that the value measured under the magnetic field shows a distinct improvement (Table 6).

Table 6 The influence of a magnetic field

\begin{tabular}{ccccc}
\hline$L / \mathrm{cm}$ & $n_{e} / \mathrm{cm}^{-3}$ & $v / \mathrm{s}^{-1}$ & $B_{0} / \mathrm{T}$ & $K / \mathrm{dB}$ \\
\hline \multirow{3}{*}{4} & \multirow{3}{*}{$3 \times 10^{13}$} & \multirow{2}{*}{$9 \times 10^{9}$} & 0.50 & 6.1 \\
& & & 0.64 & 1.6 \\
& & & 0 & 4.6 \\
& & & 0.44 & 2.8 \\
4 & \multirow{3}{*}{$4 \times 10^{12}$} & \multirow{2}{*}{$2.4 \times 10^{9}$} & 0.66 & 1.2 \\
& & & 0.74 & 1.6 \\
& & & 0.84 & 0.9 \\
\hline
\end{tabular}

\section{CONCLUSIONS}

The experimental results show that when the plasma layer is thick (such as $L / \lambda \geq 10$ ), the correlation between the attenuation coefficients of the electromagnetic waves and the parameters $n_{e}, v, \omega, L$ determined from the measurements are in good agreement with the theoretical predictions for electromagnetic wave propagations in a uniform infinite plasma. When the plasma layer is thin (such as when both $L$ and $\lambda$ are of the same order), the theoretical results are only in qualitative agreement with the experimental observations in the present parameter range, but the formula of the electromagnetic wave propagation theory in a uniform infinite plasma can not be used for quantitative computations of the correlation between the attenuation coefficients and the parameters $n_{e}, v$, $\omega, L$. In fact, if $\omega<\omega_{p}, v^{2} \ll \omega^{2}$, the power attenuations $K$ of the electromagnetic waves obtained from the measurements in the thin-layer plasma are much smaller than those of the theoretical predictions. On the other hand, if $\omega>\omega_{p}, v^{2} \ll \omega^{2}$ (just $v \approx f$ ), the measurements are much larger than the theoretical results.

Acknowledgement The author gratefully acknowledges the support of K. C. Wong Education Foundation, Hong Kong.

\section{REFERENCES}

1 Plonsey R, Collin RE. Principles and Applications of Electromagnetic Fields. New York: McGraw-Hill, 1961 
2 Friel PJ, Rosenbaum B. Propagation of electromagnetic waves through reentry-induced plasmas. $A d$ vances in the Astronautical Sciences, 1963, 11: 399

3 Bachynski MP, Gibbs BW. Propagation in plasma along the magnetic field. Phys Fluid, 1966, 9(3): 520

4 Zhu NY. Measurements of flow parameters in a $800 \mathrm{~mm}$ shock tube. Acta Mechanica Sinica, 1978, 10: $234 \sim 237$ (in Chinese)

$5 \mathrm{Zhu}$ NY. The Investigation of ionization phenomena in a $800 \mathrm{~mm}$ shock tube. In: 13th International Symposium on Shock Tubes and Waves. Niagara Falls, 1981. 528 534
6 Zhu NY. The investigation of ionization phenomena of shock tube using microwave interferometer. Acta Mechanica Sinica, 1984, 16: 85 91 (in Chinese)

7 Zhu NY. Measurements of electron density in high temperature air and inspections of theoretical charts. Journal of Chinese Society of Astronautics, 1987, (2): $86 \sim 92$ (in Chinese)

8 Zhu NY. Measurement of the average collision crosssection of slow electron using $8 \mathrm{~mm}$ microwave. In: $2 \mathrm{th}$ Symposium on Atom-Molecule and Physical Mechanics in China, 1986 